\title{
Finding Community Structure Based on Subgraph Similarity
}

\author{
Biao Xiang, En-Hong Chen, and Tao Zhou
}

\begin{abstract}
Community identification is a long-standing challenge in the modern network science, especially for very large scale networks containing millions of nodes. In this paper, we propose a new metric to quantify the structural similarity between subgraphs, based on which an algorithm for community identification is designed. Extensive empirical results on several real networks from disparate fields has demonstrated that the present algorithm can provide the same level of reliability, measure by modularity, while takes much shorter time than the well-known fast algorithm proposed by Clauset, Newman and Moore (CNM). We further propose a hybrid algorithm that can simultaneously enhance modularity and save computational time compared with the CNM algorithm.
\end{abstract}

\section{Introduction}

The study of complex networks has become a common focus of many branches of science [1]. An open problem that attracts increasing attention is the identification and analysis of communities [2]. The so-called communities can be loosely defined as distinct subsets of nodes within which they are densely connected, while sparser between which [3]. The knowledge of community structure is significant for the understanding of network evolution [4] and the dynamics taking place on networks, such as epidemic spreading [5, 6] and synchronization [7, 8]. In addition, reasonable

Biao Xiang, En-Hong Chen

Department of Computer Science, University of Science and Technology of China, Hefei Anhui 230009, P. R. China. e-mail: cheneh@ustc.edu.cn

Tao Zhou

Department of Modern Physics, University of Science and Technology of China, Hefei Anhui 230026, P. R. China, and Department of Physics, University of Fribourg, Chemin du Musée 3, Fribourg 1700, Switzerland. e-mail: zhutou@ustc.edu 
identification of communities is helpful for enhancing the accuracy of information filtering and recommendation [9].

Many algorithms for community identification have been proposed, these include the agglomerative method based on node similarity [10], divisive method via iterative removal of the edge with the highest betweenness [3, 11], divisive method based on dissimilarity index between nearest-neighboring nodes [12], a local algorithm based on edge-clustering coefficient [13], Potts model for fuzzy community detection [14], simulated annealing [15], extremal optimization [16], spectrum-based algorithm [17], iterative algorithm based on passing message [18], and so on.

Finding out the optimal division of communities, measure by modularity [11], is very hard [19], and for most cases, we can only get the near optimal division. Generally speaking, without any prior knowledge, such as the maximal community size and the number of communities, an algorithm that can give higher modularity is more time consuming [20]. As a consequence, providing accurate division of communities for a very large scale network in reasonable time is a big challenge in the modern network science. To address this issue, Newman proposed a fast greedy algorithm with time complexity $O\left(n^{2}\right)$ for sparse networks [21], where $n$ denotes the number of nodes. Furthermore, Clauset, Newman, and Moore (CNM) designed an improved algorithm giving identical result but with lower computational complexity [22], as $O\left(n \log ^{2} n\right)$. In this paper, based on a newly proposed metric of similarity between subgraphs, we design an agglomerative algorithm for community identification, which gives the same level of reliability but is typically hundreds of times faster than the CNM algorithm. We further propose a hybrid method that can simultaneously enhance modularity and save computational time compared with the CNM algorithm.

The rest of this paper is organized as follows. In Section 2, we introduce the present method, including the new metric of subgraph similarity and the corresponding algorithm, as well as the hybrid algorithm. In Section 3, we give a brief description of the empirical data used in this paper. The performance of our proposed algorithms for both algorithmic accuracy and computational time are presented in Section 4. Finally, we sum up this paper in Section 5.

\section{Method}

Considering an undirected simple network $G(V, E)$, where $V$ is the set of nodes and $E$ is the set of edges. The multiple edges and self-connections are not allowed. Denote $\Gamma=\left\{V_{1}, V_{2}, \cdots, V_{h}\right\}$ a division of $G$, that is, $V_{i} \cap V_{j}=\emptyset$ for $1 \leq i \neq j \leq h$ and $V_{1} \cup V_{2} \cup \cdots \cup V_{h}=V$. We here propose a new metric of similarity between two subgraphs, $V_{i}$ and $V_{j}$, as:

$$
s_{i j}=\frac{e_{i j}+\sum_{k=1}^{h} \frac{\sqrt{e_{i k} e_{k j}}}{\left|V_{k}\right|}}{\sqrt{d_{i} d_{j}}},
$$


where $e_{i j}$ is the number of edges with two endpoints respectively belonging to $V_{i}$ and $V_{j}\left(e_{i j}\right.$ is defined to be zero if $i=j$ ), $\left|V_{k}\right|$ is the number of nodes in subgraph $V_{k}$, and $d_{i}=\sum_{x \in V_{i}} k_{x}$ is the sum of degrees of nodes in $V_{i}$, where the degree of node $x$, namely $k_{x}$, is defined as the number of edges adjacent to $x$ in $G(V, E)$. The similarity here can be considered as a measure of proximity between subgraphs, and two subgraphs having more connections or being simultaneously closely connected to some other subgraphs are supposed to have higher proximity to each other. $d_{i}$ can be considered as the mass of a subgraph, and the denominator, $\sqrt{d_{i} d_{j}}$, is introduced to reduce the bias induced by the inequality of subgraph sizes. Note that, if each subgraph only contains a single node, as $V_{i}=\left\{v_{i}\right\}$, the similarity between too subgraphs, $V_{i}$ and $V_{j}$, is degenerated to the well-known Salton index (also called cosine similarity in the literature) [23] between $v_{i}$ and $v_{j}$ if they are not directly connected.

Our algorithm starts from an $n$-division $\Gamma_{0}=\left\{V_{1}, V_{2}, \cdots, V_{n}\right\}$ with $V_{i}=\left\{v_{i}\right\}$ for $1 \leq i \leq n$. The procedure is as follows. (i) For each subgraph $V_{i}$, let it connect to the most similar subgraphs, namely $\left\{V_{j} \mid s_{i j}=\max _{k}\left\{s_{i k}\right\}\right\}$. (ii) Merge each connected component in the network of subgraphs generated by step (i) into one subgraph, which defines the next division. (iii) Repeat the step (i) until the number of subgraphs equals one. During this procedure, we calculate the modularity for each division and the one corresponding to the maximal modularity is recorded. To make our algorithm clear to readers, we show a small scale example consisted of six subgraphs with similarity matrix:

$$
S=\left(\begin{array}{llllll}
0 & 2 & 2 & 1 & 0 & 1 \\
2 & 0 & 1 & 3 & 1 & 1 \\
2 & 1 & 0 & 1 & 0 & 1 \\
1 & 3 & 1 & 0 & 2 & 0 \\
0 & 1 & 0 & 2 & 0 & 3 \\
1 & 1 & 1 & 0 & 3 & 0
\end{array}\right) .
$$

After the step (i), as shown in Figure 1, we get a network where each node represents a subgraph. We use the directed network representation, in which a directed arc from $V_{i}$ to $V_{j}$ means $V_{j}$ is one of the most similar subgraphs to $V_{i}$. In the algorithmic implementation, those directed arcs can be treated as undirected (symmetry) edges. The network shown in Figure 1 is determined by the similarity matrix $S$, and after step (ii), the updated division contains only two subgraphs, $V_{1} \cup V_{2} \cup V_{3} \cup V_{4}$ and $V_{5} \cup V_{6}$, corresponding to the two connected components. Note that, the algorithmic procedure is deterministic and the result is therefore not sensitive to where it starts at all.

The CNM algorithm is relatively rough in the early stage, actually, it strongly tends to merge lower-degree nodes together (see Eq. (2) in Ref. [21], the first term is not distinguishable in the early stage while the enhancement of the second term favors lower-degree nodes). This tendency usually makes mistakes in the very early 


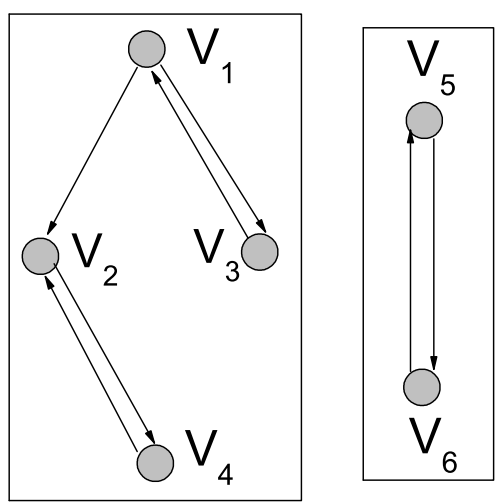

Fig. 1 Illustration of the algorithm procedure, where each node represents a subgraph. The similarities between subgraph pairs are shown in Eq. (2).

stage and can not be corrected afterwards. We therefore propose a hybrid algorithm which starts from a $n$-division $\Gamma_{0}=\left\{V_{1}, V_{2}, \cdots, V_{n}\right\}$, and takes the procedure mentioned in the last paragraph for one round (i.e., step (i) and step (ii)). The subgraph similarity is degenerated to the similarity between two nodes:

$$
s_{x y}=\frac{a_{x y}+n_{x y}}{\sqrt{k_{x} k_{y}}},
$$

where $n_{x y}$ denotes the number of common neighbors between $x$ and $y, a_{x y}$ is 1 if $x$ and $y$ are directly connected, and 0 otherwise. After this round, each subgraph has at least two nodes. Then, we implement the CNM algorithm until all nodes are merged together.

\section{Data}

In this paper, we consider five real networks drawn from disparate fields: (i) Football.- A network of American football games between Division IA colleges during regular season Fall 2000, where nodes denote football teams and edges represent regular season games [3]. (ii) Yeast PPI.-A protein-protein interaction network where each node represents a protein [24, 25]. (iii) Cond-Mat.-A network of coauthorships between scientists posting preprints on the Condensed Matter E-Print Archive from Jan 1995 to March 2005 [26]. (iv) WWW.- A sampling network of 

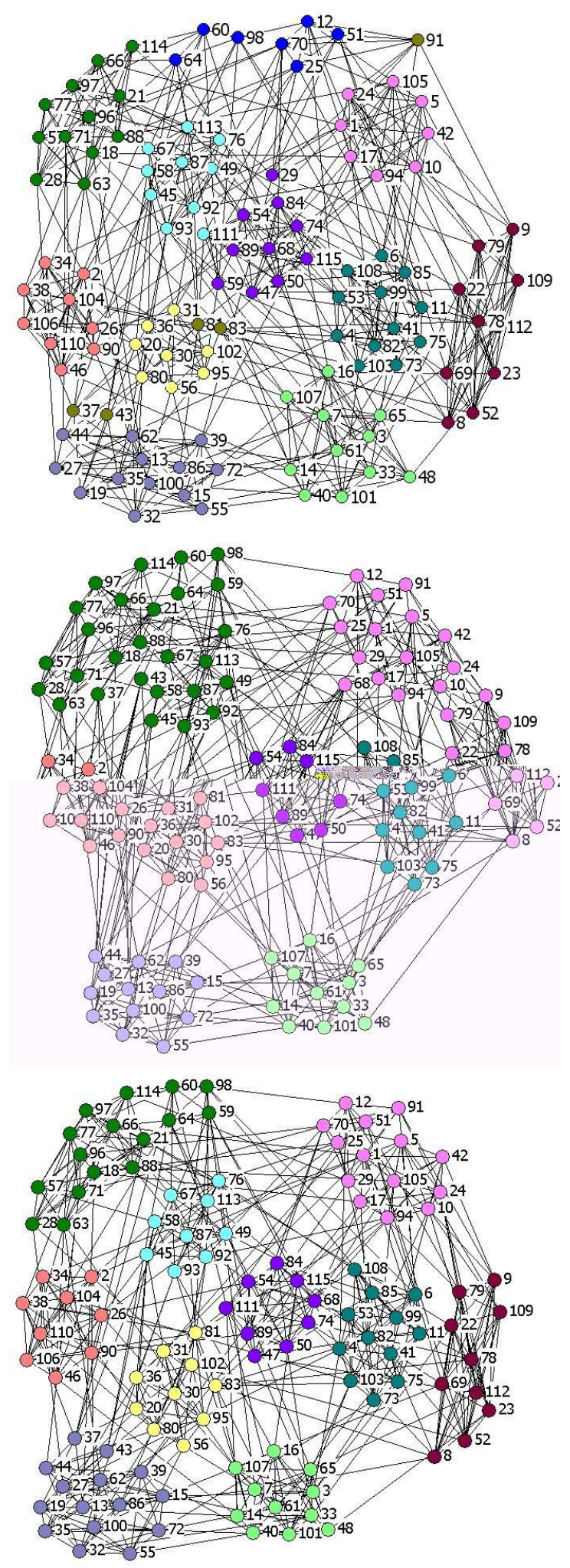

Fig. 2 Comparison of the algorithmic outputs corresponding to the best identifications subject to modularity. The three panels are (upper panel) real grouping in regular season Fall 2000, (middle panel) resulting communities from the CNM algorithm, and (lower panel) resulting communities from the $\mathrm{XCZ}+\mathrm{CNM}$ algorithm. Each node here denotes a football team and different colors represent different groups/communities. 
the World Wide Web [27]. (v) IMDB.- Actor networks from the Internet Movie Database [28]. We summarize the basic information of these networks in Table 1.

Table 1 Basic information of the networks for testing.

\begin{tabular}{llll}
\hline Networks & Number of Nodes, $|V|$ & Number of Edges, $|E|$ & References \\
\hline Football & 115 & 613 & {$[3$} \\
Yeast PPI & 2631 & 7182 & 24 \\
Cond-Mat & 40421 & 175693 & 26 \\
WWW & 325729 & 1090107 & 27 \\
IMDB & 1324748 & 3782463 & 28 \\
\hline
\end{tabular}

Table 2 Maximal modularity.

\begin{tabular}{llllll}
\hline Algorithms & Football & Yeast PPI & Cond-Mat & WWW & IMDB \\
\hline CNM & 0.577 & 0.565 & 0.645 & 0.927 & N/A \\
XCZ & 0.538 & 0.566 & 0.682 & 0.882 & 0.691 \\
XCZ+CNM & 0.605 & 0.590 & 0.716 & 0.932 & 0.786 \\
\hline
\end{tabular}

Table 3 CPU Time in millisecond (ms) resolution.

\begin{tabular}{llllll}
\hline Algorithms & Football & Yeast PPI & Cond-Mat & WWW & IMDB \\
\hline CNM & 172 & 5132 & 559781 & 12304152 & N/A \\
XCZ & 0 & 47 & 2022 & 17734 & 257875 \\
XCZ+CNM & 0 & 62 & 36422 & 443907 & 47714093 \\
\hline
\end{tabular}

\section{Result}

In Table 2 and Table 3, we respectively report the maximal modularities and the CPU times corresponding to the CNM algorithm, our proposed algorithm (referred as XCZ algorithm where XCZ is the abbreviation of the authors' names), and the hybrid algorithm (referred as XCZ+CNM). All computations were carried out in a desktop computer with a single Inter CoreE2160 processor $(1.8 \mathrm{GHz})$ and $2 \mathrm{~GB}$ EMS memory. The programme code for the CNM algorithm is directly downloaded from the personal homepage of Clauset. The IMDB seems too large for the CNM algorithm, and we can not get the result in reasonable time. 
From Table 2, one can find that the XCZ algorithm can provide competitively accurate division of communities verse the CNM algorithm. A significant feature of the $\mathrm{XCZ}$ algorithm is that it is very fast, in general more than 100 times fasters than the CNM algorithm. Just by a desktop computer, one can find out the community structure of a network containing $10^{6}$ nodes within minutes. In comparison, the hybrid algorithm is remarkably more accurate (measured by the maximal modularity) than both the CNM and XCZ algorithms. In Figure 2, we compare the resulting community structures of the Football network, from which one can see obviously that the hybrid algorithm gives closer result to the real grouping than the CNM algorithm. We think the hybrid algorithm is fast enough for many real applications. Taking IMDB as an example, although it contains more than $1.3 \times 10^{6}$ nodes, the hybrid algorithm only spends less than one day. Indeed, the hybrid algorithm outperforms the CNM algorithm for both the accuracy and the speed.

\section{Conclusion}

Thanks to the quick development of computing power and database technology, many very large scale networks, consisted of millions or more nodes, are now available to scientific community. Analysis of such networks asks for highly efficient algorithms, where the problem of community identification has attracted more and more attentions for its hardness and practical significance.

The agglomerative method based on node similarity [10] is of lower accuracy compared with the divisive algorithms based on edge-betweenness [3] and edgeclustering coefficient [13]. In this paper, we extended the similarity measuring the structural equivalence of a pair of nodes to the so-called subgraph similarity that can quantify the proximity of two subsets of nodes. Accordingly, we deigned an ultrafast algorithm, which provides competitively accurate division of communities while runs typically hundreds of times faster than the well-known CNM algorithm. Using our algorithm, just by a desktop computer, one can deal with a network of millions of nodes in minutes. For example, it takes less than five minutes to get the community structure of IMDB, which is consisted of more than $1.3 \times 10^{6}$ nodes.

Furthermore, we integrated the CNM algorithm and our proposed algorithm and designed a hybrid method. Numerical results on representative real networks showed that this hybrid algorithm is remarkably more accurate than the CNM algorithm, and can manage a network of about one million nodes in a few hours.

The modularity has been widely accepted as a standard metric for evaluating the community identification, as well as has found some other applications such as being an assistant for extracting the hierarchical organization of complex systems [29]. Although modularity is indeed the most popular metric for community identification, and the result corresponding to the maximal modularity looks very reasonable (see, for example, Figure 2), it has an intrinsic resolution limit that makes small communities hard to detect [30, 31]. An alternative, named normalized тиtual information [20] is a good candidate for future investigation. In addition, an 
extension of modularity for weighted networks, namely weighted modularity [32], has been adopted to deal with community identification problem in weighted networks [33, 34]. We hope the subgraph similarity proposed in this paper can also be properly extended to a weighted version to help extract the weighted communities.

Acknowledgements This work is benefited from the Pajek Datasets and the Internet Movie Database, as well as the network data collected by Mark Newman, Albert-László Barabási and their colleagues. E.-H.C. acknowledges the National Natural Science Foundation of China under grant numbers 60573077 and 60775037 . T.Z. acknowledges the National Natural Science Foundation of China under grant number 10635040.

\section{References}

1. M. E. J. Newman, A.-L. Barabási, D. J. Watts, The Structure and Dynamics of Networks (Princeton University Press, Princeton, 2006).

2. M. E. J. Newman, Modularity and community structure in networks, Proc. Natl. Acad. Sci. U.S.A. 103, 8577 (2006).

3. M. Girvan, M. E. J. Newman, Community structure in social and biological networks, Proc. Natl. Acad. Sci. U.S.A. 99, 7821 (2002).

4. G. Palla, A.-L. Barabási, T. Vicsek, Quantifying social group evolution, Nature 446, 664 (2007).

5. Z. Liu, B. Hu, Epidemic spreading in community networks, Europhys. Lett. 72, 315 (2005).

6. G. Yan, Z.-Q. Fu, J. Ren, W.-X. Wang, Collective synchronization induced by epidemic dynamics on complex networks with communities, Phys. Rev. E 75, 016108 (2007).

7. A. Arenas, A. Díaz-Guilera, C. J. Pérez-Vicente, Synchronization Reveals Topological Scales in Complex Networks, Phys. Rev. Lett. 96, 114102 (2006).

8. T. Zhou, M. Zhao, G.-R. Chen, G. Yan, B.-H. Wang, Phase synchronization on scale-free networks with community structure, Phys. Lett. A 368, 431 (2007).

9. G.-R. Xue, C. Lin, Q. Yang, W.-S. Xi, H.-J. Zeng, Y. Yu, Z. Chen, Scalable collaborative filtering using cluster-based smoothing, in Proceedings of the 28th Annual International ACM SIGIR conference on Research and Development in Information Retrieval (ACM Press, pp. 114-121, 2005).

10. R. L. Breiger, S. A. Boorman, P. Arabie, An algorithm for clustering relational data with applications to social network analysis and comparison with multidimensional scaling, J. Math. Psychol. 12, 328 (1975).

11. M. E. J. Newman, M. Girvan, Finding and evaluating community structure in networks, Phys. Rev. E 69, 026113 (2004).

12. H. Zhou, Distance, dissimilarity index, and network community structure, Phys. Rev. E 67, 061901 (2003).

13. F. Radicchi, C. Castellano, F. Ceccon, V. Loreto, D. Parisi, Defining and identifying communities in networks, Proc. Natl. Acad. Sci. U.S.A. 101, 2658 (2004).

14. J. Reichardt, S. Bornholdt, Detecting Fuzzy Community Structures in Complex Networks with a Potts Model, Phys. Rev. Lett. 93, 218701 (2004).

15. R. Guimerà, M. Sales, L. A. N. Amaral, Modularity from fluctuations in random graphs and complex networks, Phys. Rev. E 70, 025101 (2004)

16. J. Duch, A. Arenas, Community detection in complex networks using extremal optimization, Phys. Rev. E 72, 027104 (2005).

17. M. E. J. Newman, Finding community strcuture in networks using the eigenvectors of matrics, Phys. Rev. E 74, 036104 (2006).

18. B. J. Frey, D. Dueck, Clustering by Passing Messages Between Data Points, Science 315, 972 (2007). 
19. U. Brandes, D. Delling, M. Gaertler, R. Görke, M. Hoefer, Z. Nikoloski, D. Wagner, On Finding Graph Clusterings with Maximum Modularity, Lect. Notes Comput. Sci. 4769, 121 (2007).

20. L. Danon, A. Díaz-Guilera, J. Duch, A. Arenas, Comparing community structure identification, J. Stat. Mech. P09008 (2005).

21. M. E. J. Newman, Fast algorithm for detecting community strcuture in networks, Phys. Rev. E 69, 066133 (2004).

22. A. Clauset, M. E. J. Newman, C. Moore, Finding community structure in very large networks, Phys. Rev. E 70, 066111 (2004).

23. G. Salton, M. J. McGill, Introduction to Modern Information Retrieval (MuGraw-Hill, Auckland, 1983).

24. C. von Merging, R. Krause, B. Snel, M. Cornell, S. G. Oliver, S. Fields, P. Bork, Comparative assessment of large-scale data sets of protein-protein interactions, Nature 417, 399 (2002).

25. D. Bu, Y. Zhao, L. Cai, H. Xue, X. Zhu, H. Lu, J. Zhang, S. Sun, L. Ling, N. Zhang, G. Li, $\mathrm{R}$. Chen, Topological structure analysis of the protein-protein interaction network in budding yeast, Nucleic Acids Research 31, 2443 (2003).

26. M. E. J. Newman, The structure of scientific collaboration networks, Proc. Natl. Acad. Sci. U.S.A. 98, 404 (2001).

27. R. Albert, H. Jeong, A.-L. Barabási, Diameter of the World Wide Web, Nature 401, 130 (1999).

28. A. Ahmen, V. Batagelj, X. Fu, S.-H. Hong, D. Merrick, A. Mrvar, Visualisation and Analysis of the Internet Movie Database, in Proceedings of the 2007 Asia-Pacific Symposium on Visualization (IEEE Press, pp. 17-24, 2007).

29. M. Sales-Pardo, R. Guimerà, A. A. Moreira, L. A. N. Amaral, Extracting the hierarchical organization of complex systems, Proc. Natl. Acad. Sci. U.S.A. 104, 15224 (2007).

30. S. Fortunato, M. Bartheémy, Resolution limit in community detection, Proc. Natl. Acad. Sci. U.S.A. 104, 36 (2007).

31. A. Lancichinetti, S. Fortunato, F. Radicchi, Benchmark graphs for testing community detection algorithms, Phys. Rev. E 78, 046110 (2008).

32. M. E. J. Newman, Analysis of weighted networks, Phys. Rev. E 70, 056131 (2004).

33. Y. Fan, M. Li, P. Zhang, J. Wu, Z. Di, The effect of weight on community structure of networks, Physica A 378, 583 (2007).

34. M. Mitrović, B. Tadić, Search of Weighted Subgraphs on Complex Networks with Maximum Likelihood Methods, Lect. Notes Comput. Sci. 5102, 551 (2008). 